Proyecciones

Vol. 21, No 3, pp. 261-276, December 2002.

Universidad Católica del Norte

Antofagasta - Chile

\title{
CRITICAL POINT THEOREMS AND APPLICATIONS
}

\author{
HAFIDA BOUKHRISSE \\ and \\ MIMOUN MOUSSAOUI \\ University Mohamed I, Morocco
}

\begin{abstract}
We Consider the nonlinear Dirichlet problem:

(1) $\left\{\begin{aligned}-\Delta u & =D_{u} F(x, u)+\lambda_{k} u & & \text { dans } \Omega \\ u & =0 & & \text { sur } \partial \Omega\end{aligned}\right.$
\end{abstract}

where $\Omega \in R^{N}$ is a bounded open domain, $F: \Omega \times R \rightarrow R$ is a carathéodory function and $D_{u} F(x, u)$ is the partial derivative of $F$. We are interested in the resolution of problem (1) when $F$ is concave. Our tool is absolutely variational. Therefore, we state and prove a critical point theorem which generalizes many other results in the literature and leads to the resolution of problem (1). Our theorem allows us to express our assumptions on the nonlinearity in terms of $F$ and not of $\nabla F$. Also, we note that our theorem doesn't necessitate the verification of the famous compactness condition introduced by Palais-Smale or any of its variants.

Key words: Critical point theory, convexity conditions, Elliptic semilinear problem. 


\section{Introduction}

We Consider the nonlinear Dirichlet problem:

$$
\text { (1) }\left\{\begin{aligned}
-\Delta u & =D_{u} F(x, u)+\lambda_{k} u & & \operatorname{dans} \Omega \\
u & =0 & & \operatorname{sur} \partial \Omega
\end{aligned}\right.
$$

where $\Omega \in R^{N}$ is a bounded open domain, $F: \Omega \times R \rightarrow R$ is a carathéodory function and $D_{u} F(x, u)$ is the partial derivative of $\mathrm{F}$. We assume: $D_{u} F(x, u)=f(x, u)$. The spectrum of $(-\Delta)$ is denoted as

$$
0<\lambda_{1}<\lambda_{2}<\ldots<\lambda_{k}<\lambda_{k+1}<\ldots
$$

The aim of this paper is to resolve the problem $(\mathrm{P})$ with variational tool. We suppose that $\mathrm{F}$ satisfies some growth conditions and some classical assymptotic assumptions. Therefore, it turns out that the weak solutions of (1) are precisely the critical points of the functional $\Phi: E \rightarrow R$ of the form

$$
\Phi(u)=\frac{1}{2}\|u\|^{2}-f(x, u) .
$$

We restrict ourselves to the case where $\mathrm{F}$ is concave. In contrast with classical critical point theorems, we state and prove a critical point theorem which allows us to express our assumptions on the nonlinearity in terms of $\mathrm{F}$ and not of $\nabla F$ in the sense of (2) below. Also, we note that our theorem doesn't necessitate the verification of the famous compactness condition introduced by Palais-smale in [1] or any of its variants, see [2] and [3]. The problem (1) is equivalent to the problem

$$
\text { (2) }\left\{\begin{aligned}
-\Delta u & =D_{u} G(x, u) & & \text { dans } \Omega \\
u & =0 & & \text { sur } \partial \Omega
\end{aligned}\right.
$$

where $G(x, u)=F(x, u)+\frac{\lambda_{k}}{2} u^{2}$ and $D_{u} G(x, u)=g(x, u)$. To resolve problem (2), the classical results considered the case when the following quotients

$$
\frac{2 G(x, s)}{s^{2}} \quad \text { and } \quad \frac{g(x, s)}{s}
$$

are situated between two successive eigenvalues $\lambda_{k}$ and $\lambda_{k+1}$.

This kind of problems has been studied a long time ago. On 1930 Hammerstein in [4] proved a result of existence for (2) if $\mathrm{f}$ is continuous and satisfies a linear growth condition and

$$
\limsup _{|s| \rightarrow \infty} \frac{2 G(x, s)}{s^{2}} \leq \mu, \quad \mu<\lambda_{1} .
$$


On 1949, in [5], Dolph proved the first result of existence when

$$
\lambda_{k}<\mu_{k} \leq \liminf _{|s| \rightarrow \infty} \frac{g(x, s)}{s} \leq \limsup _{|s| \rightarrow \infty} \frac{g(x, s)}{s} \leq \mu_{k+1}<\lambda_{k+1} .
$$

where $\lambda_{k}$ and $\lambda_{k+1}$ are two consecutive eigenvalues of $-(\Delta)$ in $H_{0}^{1}(\Omega)$. Then, Dolph considered the last condition with $\mathrm{G}$ instead of g:

$$
\lambda_{k}<\mu_{k} \leq \liminf _{|s| \rightarrow \infty} \frac{G(x, s)}{s} \leq \limsup _{|s| \rightarrow \infty} \frac{G(x, s)}{s} \leq \mu_{k+1}<\lambda_{k+1} .
$$

The first variational attempt to solve problem (2) under condition (2) was carried over by Dolph, see [6]. He assumed in addition the following condition: Denoting by $V=\oplus_{i \leq k} E\left(\lambda_{i}\right)$ where $E\left(\lambda_{i}\right)$ is the eigenspace associated to the eigenvalue $\lambda_{i}$ and by $\mathrm{W}$ its orthogonal so that: $H_{0}^{1}=V \oplus W$, and we denote $V_{w}=w+V$ with $w \in W$.

The functional associated to problem (2): $\Phi(u)=\frac{1}{2} \int_{\Omega}\|\nabla u\|^{2}-\int_{\Omega} G(x, u) d x$ admits at most one maximum in $w+V$ for all $w \in W$.

Our absract theorem generalizes a Mini-Max theorem due to Lazer et al. [9] to the case where $\mathrm{X}$ and $\mathrm{Y}$ are not necessarily finite dimensional. Moreover, we consider $\Phi$ of class $\mathcal{C}^{1}$ instead of $\mathcal{C}^{2}$. Tersian [10] studied the case where $\mathrm{X}$ and $\mathrm{Y}$ are not necessarily finite dimensional and $\nabla \Phi: H \rightarrow H$ is everywhere defined and hemicontinuous on $\mathrm{H}$, which means that

$$
\lim _{t \rightarrow 0} \nabla \Phi(u+t v)=\nabla \Phi(u) \quad \forall u, v \in H .
$$

Instead of conditions on Hessian of $\Phi$, they supposed

$(1)\left(\nabla \Phi\left(h_{1}+y\right)-\nabla \Phi\left(h_{2}+y\right), h_{1}-h_{2}\right) \leq-m_{1}\left\|h_{1}-h_{2}\right\|^{2} \quad h_{1}, h_{2} \in X, \quad y \in$ $Y$,

$(2)\left(\nabla \Phi\left(x+k_{1}\right)-\nabla \Phi\left(x+k_{2}\right), k_{1}-k_{2}\right) \geq m_{2}\left\|k_{1}-k_{2}\right\|^{2} \quad k_{1}, k_{2} \in Y, \quad x \in X$, where $H=X \oplus Y, m_{1}$ and $m_{2}$ are strictly positives.

Their result rests heavily upon two theorems on $\alpha$-convex functionals and an existence theorem for a class of monotone operators due to Browder. Our theorem partially extend many other results in the literature (see e.g.[11] and [12]). On 1991, the second author of this paper proved in [13] the following theorem. 
Theorem 1.1. Let $H$ be a Hilbert space such that : $H=V \oplus W$ where $V$ is finite dimensional subspace of $H$ and $W$ its orthogonal. Let $\Phi: H \rightarrow R$ a functional such that:

(i) $\Phi$ is of class $\mathcal{C}^{1}$.

(ii) $\Phi$ is coercive on $W$ i.e, $\Phi(w) \rightarrow+\infty$ when $\|w\| \rightarrow+\infty$.

(iii) For fixed $w \in W, v \mapsto \Phi(v+w)$ is concave on $V$.

(iv) For fixed $w \in W, \Phi(v+w) \rightarrow-\infty$ when $\|v\| \rightarrow+\infty, v \in V$; and the convergence is uniform on bounded subsets of $W$.

(v) for all $v \in V, \Phi$ is weakly lower semicontinuous on $W+v$.

Then $\Phi$ admits a critical point in $H$.

In our theorem, we are based specially on theorem 1.1. We note that our theorem generalizes theorem 1.1 and our previous result [14] and our convexity conditions are weaker than all used in the previous results.

In [13], Moussaoui resolved problem (1) by theorem 2.1 with the following assumptions

(F1) $F(x,$.$) is convex and differentiable for almost every x \in \Omega$.

$F(., s)$ is measurable for all $s \in R$.

(F2) $\forall r>0 \quad \sup _{|s| \leq r}|F(x, s)| \in L^{1}(\Omega)$.

(F3) There exist $l, \beta \in L^{2}(\omega), \beta \geq 0$ such that

$$
F(x, s) \geq \operatorname{sl}(x)-\beta(x) .
$$

(F4) $\lim \sup _{|s| \rightarrow+\infty} \frac{2 F(x, s)}{s^{2}}=\alpha(x) \leq \lambda_{k+1}-\lambda_{k}$.

with $\alpha(x) \geq 0$ and $\left\{x \in \Omega \quad \alpha(x)<\lambda_{k+1}-\lambda_{k}\right\}$ is of positive measure.

(F5) $\int_{\Omega} F\left(x, v^{0}\right) d x \rightarrow+\infty$ when $\left\|v^{0}\right\| \rightarrow+\infty, v^{0} \in E\left(\lambda_{k}\right)$.

The result obtained by Moussaoui is a particular case of Mawhin and Willem result, see [15]. Or the proof used by Moussaoui is absolutely different from the proof used by Mawhin and Willem. In fact, Mawhin and Willem used the dual least action principle of Clarke-Ekeland which is essentially a convex analysis method to solve a perturbed problem. They combined this process to an approximation dealing to attain their aim.

For us, we will be interested te resolve problem (1) with "dual" conditions of those supposed by Moussaoui and we note that our resolution of problem (1) doesn't require the condition of Ahmed,Lazer and Paul (see $[16])$. 


\section{Critical point theorems}

\section{The main result.}

Theorem 2.1. Let $E$ be a Hilbert space such that: $E=V \oplus W$ where $V$ and $W$ are two closed subspaces of $E$. Let $\Phi: E \rightarrow R$ a functional such that:

(i) $\Phi$ is of class $\mathcal{C}^{1}$.

(ii) For each $w \in W, \Phi$ is coercive on $V+w$. i.e, $\Phi(v+w) \rightarrow+\infty$ when $\|v\| \rightarrow+\infty$.

(iii) There exists an increasing function $\gamma:(0,+\infty) \rightarrow(0,+\infty)$ such that:

$$
\left(\nabla \Phi\left(w+v_{1}\right)-\nabla \Phi\left(w+v_{2}\right), v_{1}-v_{2}\right) \geq \gamma\left(\left\|v_{1}-v_{2}\right\|\right)\left\|v_{1}-v_{2}\right\|,
$$

for all $v_{1}, v_{2}$ in $V$ and $w$ in $W$.

(iv) $\Phi$ is anticoercive on $W$. i.e, $\Phi(w) \rightarrow-\infty$ when $\|w\| \rightarrow+\infty$.

(v) For all $v \in V, \Phi$ is weakly upper semi-continuous on $W+v$.

Then $\Phi$ admits at least a critical point $u \in H$. Moreover, this critical point is characterized by the equality

$$
\Phi(u)=\max _{w \in W} \min _{v \in V} \Phi(v+w) .
$$

Remark 2.2. Condition (ii) in theorem 2.1 doesn't contain the " uniform convergence on bounded subsets of $W$ " which is essential in theorem 1.1. This elimination will permit us to improve the resolution of problème (1). In fact we will solve problem (1) (paragraph 4) without Ahmed,lazer and Paul condition (A.L.P) which was used in many works (see e.g.[15]and [13] ) .

The proof of theorem 2.1 will depend on three lemmas. Lemmas 3.4 and 2.4 were used by Moussaoui to prove the existence of critical points of $\Phi$ when $\mathrm{V}$ is of finite dimension (theorem 1.1, introduction).

Lemma 2.3. For all $w \in W$, there exists a unique $v \in V$ such that :

$$
\Phi(v+w)=\max _{g \in V} \Phi(g+w) .
$$

Proof: From (iii), for $w$ fixed in $W, v \mapsto \Phi(v+w)$ is continuous and strictly convex on $\mathrm{V}$. Then, it is weakly lower semicontinuous on $V$. Moreover, from (ii), it is coercive on V. So that, from a lemma in [17] and [18] for 
axample, it admits a minimum on $V$. We affirm that this minimum is unique, otherwise we suppose that there exist two minimums $v_{1}$ and $v_{2}$. Let $v_{\lambda}=\lambda v_{1}+(1-\lambda) v_{2} \quad$ for $\quad 0<\lambda<1$, then

$$
\Phi\left(v_{\lambda}+w\right)<\lambda \Phi\left(v_{1}+w\right)+(1-\lambda) \Phi\left(v_{2}+w\right)=\Phi\left(v_{1}+w\right)=\Phi\left(v_{2}+w\right) .
$$

In the proof of this theorem, we will adopt the notations:

$$
\begin{gathered}
\bar{V}(w)=\left\{v \in V: \Phi(v+w)=\min _{g \in V} \Phi(g+w)\right\} \\
S=\{u=v+w, \quad w \in W, \quad v \in \bar{V}(w)\} .
\end{gathered}
$$

and

$$
S_{w}=\{u=v+w, \quad v \in \bar{V}(w)\} .
$$

Lemma 2.4. There exists $u \in S$ such that

$$
\Phi(u)=\max _{S} \Phi
$$

Proof: There exists a sequence $\left(u_{n}\right)$ of S such that $\Phi\left(u_{n}\right) \rightarrow \sup _{S} \Phi=a$. For each $n, u_{n}=v_{n}+w_{n}$ with $w_{n} \in W$ and $v_{n} \in V\left(w_{n}\right)$.

Claim

$$
\left\|w_{n}\right\| \leq c
$$

Otherwise

$$
\Phi\left(u_{n}\right)=\Phi\left(v_{n}+w_{n}\right) \leq \Phi\left(w_{n}\right) .
$$

From (iv), $\Phi\left(w_{n}\right) \rightarrow-\infty$, hence $\Phi\left(u_{n}\right) \rightarrow-\infty$. A contradiction. From (5), there exists a subsequence denoted also $w_{n}$ such that $w_{n} \rightarrow w$. Take $\mathrm{v}$ in $V$, by (v), we have:

$$
\Phi(v+w) \geq \liminf _{n} \Phi\left(v+w_{n}\right) \geq \liminf _{n} \Phi\left(v_{n}+w_{n}\right)=a
$$

This is true for all $v \in V$, in particular for $v \in V(w)$. Then $u=v+w$ satisfies (4).

Lemma 2.5. The application $f: W \rightarrow V$ such that

$$
\Phi(w+f(w))=\min _{g \in V} \Phi(g+w)
$$

is continuous. 
Proof: We suppose that $\mathrm{f}$ isn't continuous, thus there exist $\delta>0$ and a sequence $\left(w_{n}\right)$ converging to $w \in W$ such that for enough big n, we have:

$$
\left\|f\left(w_{n}\right)-f(w)\right\| \geq \delta .
$$

From (ii), it is clear that

$$
(\nabla \Phi(w+f(w)), v)=0 \quad \forall v \in V .
$$

Let $\mathrm{P}$ be the projection of $\mathrm{H}$ onto $\mathrm{V}$ defined by $P(v+w)=v$, and let $P^{*}$ be the adjoint of $\mathrm{P}$. Then we obtain for each $\mathrm{n}$ :

$$
\begin{array}{r}
\left\|P^{*}\left(\nabla \Phi\left(w_{n}+f(w)\right)\right)\right\|\left\|f\left(w_{n}\right)-f(w)\right\| \\
\geq-\left(\nabla \Phi\left(w_{n}+f(w)\right), f\left(w_{n}\right)-f(w)\right) \\
\geq\left(\nabla \Phi\left(w_{n}+f\left(w_{n}\right)\right)-\nabla \Phi\left(w_{n}+f(w)\right)\right. \\
\left.f\left(w_{n}\right)-f(w)\right) \\
\geq \gamma\left(\left\|f\left(w_{n}\right)-f(w)\right\|\right)\left\|f\left(w_{n}\right)-f(w)\right\| .
\end{array}
$$

Hence, from (4) and (iii), we conclude that for enough big n, there exists $a>0$ such that

$$
\left\|P^{*}\left(\nabla \Phi\left(w_{n}+f(w)\right)\right)\right\| \geq \gamma\left(\left\|f\left(w_{n}\right)-f(w)\right\|\right) \geq a .
$$

In the other hand,

$$
\nabla \Phi\left(w_{n}+f(w)\right) \rightarrow \nabla \Phi(w+f(w)) .
$$

Thus

$$
\left(\nabla \Phi\left(w_{n}+f(w)\right), v\right) \rightarrow 0
$$

for each $\mathrm{v}$ in $\mathrm{V}$. We conclude that

$$
\left\|P^{*}\left(\nabla \Phi\left(w_{n}+f(w)\right)\right)\right\| \rightarrow 0 .
$$

A contradiction.

\section{Proof of theorem 2.1.}

Let $w \in W$ and $u \in S_{w}$. We will prove that if $\mathrm{u}$ satisfies (4), then $\mathrm{u}$ is a critical point of $\Phi$.

From lemma 3.4, we have $(\nabla \Phi(u), v)=0 \quad$ for all $\mathrm{v}$ in $\mathrm{V}$, so it suffices to prove that

$$
(\nabla \Phi(u), h)=0 \quad \forall h \in W
$$


Recall that $u \in S_{w}$ is written: $u=v+w$ where $w \in W$ and $v \in \bar{V}(w)$. Take $h \in W$ and $w_{t}=w+t h$ for $|t| \leq 1$. For each $\mathrm{t}$ such that $0<|t| \leq 1$, there exists a unique $v_{t_{n}} \in \bar{V}\left(w_{t_{n}}\right)$. Since $w_{t_{n}} \rightarrow w$ when $n \rightarrow+\infty$, we deduce by lemma 2.4 that $v_{t_{n}}$ converges to a certain $v_{0}$ and $v_{0} \in \bar{V}(w)$. Then, by lemma 3.4, we conclude that $v_{0}=v$. For $t>0$, since $v_{0}+w \in S_{w}$ we have

$$
\frac{\Phi\left(w_{t}+v_{t}\right)-\Phi\left(v_{t}+w\right)}{t} \geq \frac{\Phi\left(w_{t}+v_{t}\right)-\Phi\left(v_{0}+w\right)}{t} \geq 0 .
$$

Then,

$$
\left(\nabla \Phi\left(v_{t}+w+\lambda_{t} t h\right), h\right) \geq 0 \quad 0<\lambda_{t}<1 .
$$

At the limit, we obtain

$$
(\nabla \Phi(u), h)=0 \quad \forall h \in W
$$

Then, $\mathrm{u}$ is a critical point of $\Phi$.

\section{Variants of theorem 2.1}

The first theorem that we will present is the "dual" version of theorem 1.1.

Theorem 3.1. Let $H$ be a Hilbert space such that: $H=V \oplus W$ where $V$ and $W$ are two closed subspaces of $H$. Let $\Phi: H \rightarrow R$ a functional such that:

(i) $\Phi$ is of class $\mathcal{C}^{1}$.

(ii) For all $w \in W, \Phi$ is anticoercive on $V+w$. i.e, $\Phi(v+w) \rightarrow-\infty$ when $\|v\| \rightarrow+\infty$.

(iii) There exists an increasing function $\gamma:(0,+\infty) \rightarrow(0,+\infty)$ such that:

$$
\left(\nabla \Phi\left(w+v_{1}\right)-\nabla \Phi\left(w+v_{2}\right), v_{1}-v_{2}\right\rangle \leq-\gamma\left(\left\|v_{1}-v_{2}\right\|\right)\left\|v_{1}-v_{2}\right\|,
$$

for all $v_{1}, v_{2}$ in $V$ and $w$ in $W$.

(iv) $\Phi$ is coercive on $W$. i.e, $\Phi(w) \rightarrow+\infty$ when $\|w\| \rightarrow+\infty$.

(v) For all $v \in V, \Phi$ is weakly lower semi-continuous on $W+v$.

Then $\Phi$ admits at least a critical point $u \in H$. Moreover, this critical point is characterized by the equality

$$
\Phi(u)=\min _{w \in W} \max _{v \in V} \Phi(v+w)
$$


The proof of theorem 3.1 is made by a dual manner of the proof of theorem 2.1.

Now, before announcing the second variant of theorem 3.1, We will give some definitions.

- Let $\mathrm{A}$ a convex set. The function $f: A \rightarrow R$ is quasiconcave if for all $x_{1}, x_{2}$ in $\mathrm{A}$, and for all $\lambda$ in $] 0,1[$, we have

$$
f\left(\lambda x_{1}+(1-\lambda) x_{2}\right) \geq \min \left(f\left(x_{1}\right), f\left(x_{2}\right)\right) .
$$

- The function $\mathrm{f}$ is quasiconvex if (-f) is quasiconcave, and it is strictly quasiconcave if the inequality above is strict.

- It is clear that any strictly concave function is strictly quasiconcave.

Proposition 3.2. Let $E$ be a reflexive Banach space. If $\Phi: E \rightarrow R$ is quasiconvex and lower semicontinuous, then $\Phi$ is weakly lower semicontinuous.

Theorem 3.3. Let $E$ be a reflexive Banach space such that: $E=V \oplus W$ where $V$ and $W$ are two closed subspaces of $E$. Let $\Phi: E \rightarrow R$ a functional such that:

(i) $\Phi$ is of class $\mathcal{C}^{1}$.

(ii) $\Phi^{\prime}$ is weakly continuous from $E$ to $E$.

(iii)For each $w \in W, \Phi(v+w) \rightarrow+\infty$ when $\|v\| \rightarrow+\infty$ and the convergence is uniform on bounded subsets of $W$.

(iv) For each $w \in W, \Phi$ is strictly quasiconvex on $V+w$.

(v) $\Phi$ is anticoercive on $W$. i.e, $\Phi(w) \rightarrow-\infty$ when $\|w\| \rightarrow+\infty$

(vi) For each $v \in V, \Phi$ is weakly upper semi-continuous on $W+v$.

Then $\Phi$ admits at least a critical point $u \in H$. Moreover, this critical point is characterized by the equality:

$$
\Phi(u)=\max _{w \in W} \min _{v \in V} \Phi(v+w) .
$$

\section{Proof of theorem 3.3}

The structure of the proof of theorem 3.3 is the same as theorem 2.1. So, we will report only the changes wich concern lemmas 3.4 and 2.5.

Lemma 3.4. For all $w \in W$, there exists a unique $v \in V$ such that :

$$
\Phi(v+w)=\min _{g \in V} \Phi(g+w) .
$$


Proof. For each w in $W, v \mapsto \Phi(v+w)$ is continuous and quasiconvex on V. Thus, by propsition 3.2, it's weakly lower semi-continuous on $V$. Moreover, from (iii), it's coercive, then it admits a minimum on the reflexive Banach space $\mathrm{V}$. Since $v \mapsto \Phi(v+w)$ is strictly quasiconvex, this minimum is unique. Otherwise, there exist two minimums $v_{1}$ and $v_{2}$. Let $v_{\lambda}=\lambda v_{1}+(1-\lambda) v_{2}$ for $0<\lambda<1$, then

$$
\Phi\left(v_{\lambda}+w\right)>\max \left(\Phi\left(v_{1}+w\right), \Phi\left(v_{2}+w\right)\right)=\Phi\left(v_{1}+w\right)=\Phi\left(v_{2}+w\right) .
$$

Absurde.

Lemma 3.5. The application $f: W \rightarrow V$ such that

$$
\Phi(w+f(w))=\min _{g \in V} \Phi(g+w)
$$

is continuous.

Proof. First, we prove that the application (f) defined in lemma 3.5 is bounded on bounded sets of W. Let $M>0$, from (v) and (vi) of theorem 4.1 we conclude that $\Phi$ admits a maximum on $\mathrm{W}$, so there exists a constant $N>0$ such that $\Phi(w) \leq N$ for $w \in W$ and $\|w\| \leq M$. By (iii), there exists a constante $\delta>0$ such that for $v \in V, w \in W,\|w\| \leq M$ and $\|v\| \geq \delta$, we have $\Phi(v+w) \geq 2 N$.

Since $\Phi(w+f(w))=\min _{g \in V} \Phi(w+g) \leq \phi(w)<N$, we conclude that $\|f(w)\| \leq \delta$. Next, we prove that (f) is continuous. Suppose that $w_{n}$ converges to $\mathrm{w}$, thus $f\left(w_{n}\right)$ is bounded, so there exists a subsequence of $f\left(w_{n}\right)$ denoted also $f\left(w_{n}\right)$ which converges weakly to $v_{0}$. Since $\Phi^{\prime}$ is weakly continuous, we obtain

$$
\left\langle\phi^{\prime}\left(w_{n}+f\left(w_{n}\right)\right), v\right\rangle \rightarrow\left\langle\phi^{\prime}\left(w+v_{0}\right), v\right\rangle \quad \forall v \in V .
$$

Since $\left\langle\phi^{\prime}\left(w_{n}+f\left(w_{n}\right), v\right\rangle=0\right.$ and $f(w)$ is the unique element of $\mathrm{V}$ such that $\left\langle\phi^{\prime}(w+f(w)), v\right\rangle=0$ for all $v \in V$, we conclude that $v_{0}=f(w)$.

Remark 3.6. Theorem 3.3 is a complete generalization of theorem (3.7) in [14] because it deals with a more general class of functionals than the second theorem. 


\section{Application}

We Consider the nonlinear Dirichlet problem:

$$
\text { (1) }\left\{\begin{aligned}
-\Delta u & =\lambda_{k} u+D_{u} F(x, u) & & \operatorname{dans} \Omega \\
u & =0 & & \operatorname{sur} \partial \Omega
\end{aligned}\right.
$$

where $\Omega \in R^{N}$ is a bounded open domain, $F: \Omega \times R \rightarrow R$ is a carathéodory function and $D_{u} F(x, u)$ is the partial derivative of $\mathrm{F}$ par rapport à u. We assume:

(F1) $F(x, s)$ is concave and of class $\mathcal{C}^{1}$ for almost every $x \in \Omega$.

$\mathrm{F}(., \mathrm{s})$ is measurable for all $s \in R^{N}$

(F2) There exist $a>0$ and $\beta(x) \in L^{p^{\prime}}(\Omega)$ such that

$$
\left|D_{u} F(x, u)\right| \leq a|u|^{p-1}+\beta(x) .
$$

with $1 \leq p<2^{*}$ si $N \geq 3 \quad$ et $\quad 1 \leq p<\infty \quad$ si $N=1,2$.

(F3) $\liminf _{\|s\| \rightarrow+\infty} \frac{2 F(x, s)}{s^{2}}=\alpha(x) \geq 0$

with $\{x \in \Omega: \alpha(x)>0\}$ is of positive measure.

Theorem 4.1. If $F$ satisfies $(F 1),(F 2),(F 3)$ and $(F 4)$ then the problem (1) admits at least a solution.

Proof of theorem

Denote by $\Phi$ the functional associated to problem (1)

$$
\Phi(u)=q(u)-\int_{\Omega} F(x, u) d x \quad \text { where } \quad q(u)=\frac{1}{2}\|u\|^{2}-\frac{\lambda_{k}}{2}\|u\|_{2}^{2}
$$

We note that $H_{0}^{1}(\Omega)=V \oplus W$ where $V=\oplus_{i \leq k} E\left(\lambda_{i}\right)$ and $W=V^{\perp}=$ $\oplus_{i \geq k+1} E\left(\lambda_{i}\right)$.

The critical point of $\Phi$ is the weak solution of problem (1). So, for the proof of therem 4.1, we will verifie successively hypotheses (i) to (v) of theorem 3.1 .

(i) From $(\mathrm{F} 2), \Phi$ is of class $\left(\mathcal{C}^{1}\right)$ on $H_{0}^{1}(\Omega)$

(ii) We show that $\Phi$ is coercive on $W+v$ for all $v \in V$. We have

$$
\Phi(v+w)=\frac{1}{2}\|w\|^{2}-\frac{\lambda_{k}}{2}\|w\|_{2}^{2}+\frac{1}{2}\|v\|^{2}-\frac{\lambda_{k}}{2}\|v\|_{2}^{2}-\int F(v+w) .
$$


Then there exists $c_{1}$ in $R$ such that

$$
\Phi(v+w)=\frac{1}{2}\|w\|^{2}-\frac{\lambda_{k}}{2}\|w\|_{2}^{2}-\int F(v+w)+c_{1} .
$$

Since F is concave, we conclude

$$
F\left(\frac{w}{2}\right)=F\left(\frac{v+w}{2}-\frac{v}{2}\right) \geq \frac{1}{2} F(v+w)+\frac{1}{2} F(-v) .
$$

Hence

$$
-F(v+w) \geq F(-v)-2 F\left(\frac{w}{2}\right)
$$

Since the roles of $\mathrm{V}$ and $\mathrm{W}$ are symetriques, we obtain

$$
-F(v+w) \geq F(-w)-2 F\left(\frac{v}{2}\right) .
$$

From (F3), $\forall \varepsilon>0, \exists \beta \in L^{1}(\Omega)$ such that

$$
F(x, s) \geq \frac{-\varepsilon}{2} s^{2}-\beta(x) .
$$

Thus, we obtain

$$
\Phi(v+w) \geq \frac{1}{2}\|w\|^{2}-\frac{\lambda_{k}}{2}\|w\|_{2}^{2}-\frac{\varepsilon}{2}\|w\|_{2}^{2}+c_{2} .
$$

Since $\|w\|^{2} \geq \lambda_{k+1}\|w\|_{2}^{2}, \forall w \in W$, we have

$$
\Phi(v+w) \geq \frac{1}{2}\left(1-\frac{\lambda_{k}+\varepsilon}{\lambda_{k+1}}\right)\|w\|^{2}+c_{2} .
$$

We choise $\varepsilon$ such that $\varepsilon<\lambda_{k+1}-\lambda_{k}$. Hence

$$
\Phi(v+w) \rightarrow+\infty \quad \text { si } \quad\|w\| \rightarrow+\infty .
$$

(iii) We have

$$
(\nabla \Phi(u), v)=(L u, v)-\left(D_{u} F(u), v\right),
$$

with $L(u)=-\Delta u-\lambda_{k} u$ and $u, v \in H_{0}^{1}$. So that, for $v \in V$ and $w_{1}, w_{2} \in W$, we have

$$
\begin{array}{r}
\left(\nabla \Phi\left(v+w_{1}\right)-\nabla \Phi\left(v+w_{2}\right), w_{1}-w_{2}\right)= \\
\left(L\left(w_{1}-w_{2}\right), w_{1}-w_{2}\right)+ \\
\left(D_{v+w_{2}} F\left(., v+w_{2}\right)-D_{v+w_{1}} F\left(., v+w_{1}\right), w_{1}-w_{2}\right)
\end{array}
$$


Since $F(x,$.$) is concave for all x \in \Omega$, then $-\nabla F(x,$.$) is monotone for all$ $x \in \Omega$. So

$$
\left(\nabla F\left(x, v+w_{2}\right)-\nabla F\left(x, v+w_{1}\right), w_{2}-w_{1}\right) \leq 0
$$

for all $w_{1}, w_{2} \in W, v \in V$ et $x \in \Omega$. Then

$$
\left(\nabla \Phi\left(v+w_{1}\right)-\nabla \Phi\left(v+w_{2}\right), w_{1}-w_{2}\right) \geq\left(L\left(w_{1}-w_{2}\right), w_{1}-w_{2}\right) .
$$

In the other hand, we have $\left(L\left(w_{1}-w_{2}\right), w_{1}-w_{2}\right)=\frac{1}{2}\left\|w_{1}-w_{2}\right\|^{2}-\frac{\lambda_{k}}{2} \| w_{1}-$ $w_{2} \|_{2}^{2}$ and $\left\|w_{1}-w_{2}\right\|^{2} \geq \lambda_{k+1}\left\|w_{1}-w_{2}\right\|_{2}^{2}$, so

$$
\left\|w_{1}-w_{2}\right\|_{2}^{2} \leq \frac{1}{\lambda_{k+1}}\left\|w_{1}-w_{2}\right\|^{2} .
$$

Then

$$
\left(L\left(w_{1}-w_{2}\right), w_{1}-w_{2}\right) \geq \frac{1}{2}\left(1-\frac{\lambda_{k}}{\lambda_{k+1}}\right)\left\|w_{1}-w_{2}\right\|^{2} .
$$

We conclude that there exists $a=\frac{1}{2}\left(1-\frac{\lambda_{k}}{\lambda_{k+1}}\right)>0$ such that

$$
\left(\nabla \Phi\left(v+w_{1}\right)-\nabla \Phi\left(v+w_{2}\right), w_{1}-w_{2}\right) \geq a\left\|w_{1}-w_{2}\right\|^{2} .
$$

(iv) We show that $\Phi$ is anticoercive on $\mathrm{V}$.

Claim: From (F4), there exists $\delta<0$ such that

$$
\rho(v)=\|v\|^{2}-\lambda_{k}\|v\|_{2}^{2}-\int_{\Omega} \alpha(x) v^{2} \leq \delta\|v\|^{2}, \quad \forall v \in V
$$

So we have

$$
\rho(v) \leq\|v\|^{2}-\lambda_{k}\|v\|_{2}^{2} \leq 0 \quad \forall v \in V
$$

If $\rho(v)=0$, we obtain:

$$
0=\rho(v) \leq\|v\|^{2}-\lambda_{k}\|v\|_{2}^{2} \leq 0
$$

hence $\|v\|^{2}=\lambda_{k}\|v\|_{2}^{2}$, so that $\mathrm{v}$ is an eigenvector associated to $\lambda_{k}$. Or

$$
0=\rho(w)=\int_{\Omega}-\alpha(x) v^{2}=0
$$

implies that $v(x)=0$ when $\alpha(x)>0$, so $v=0$ We suppose that the claim is false, then there exist a sequence $v_{n}$ such that $\left\|v_{n}\right\|^{2}=1$ and $v_{n} \rightarrow v\left(v_{n}\right.$ converges weakly in $\mathrm{V}), v_{n} \rightarrow v$ strongly in $L^{2}(\Omega)$ and $0 \geq \rho\left(v_{n}\right) \rightarrow 0, n \rightarrow$ $\infty$. We have

$$
\rho(v) \geq \limsup _{n} \rho\left(v_{n}\right)=0
$$


Hence $v=0$, so $v_{n} \rightarrow 0$ strongly in $L^{2}(\Omega)$ and

$$
\rho\left(v_{n}\right)=1-\lambda_{k} \int_{\Omega} v_{n}^{2}-\int_{\Omega} \alpha(x) v_{n}^{2} \rightarrow 1
$$

which contradicts the fact that $\rho\left(w_{n}\right) \rightarrow 0$, and so the claim is proved.

On the other hand, $\forall \varepsilon>0, \exists M>0$ such that

$$
F(x, s) \geq \frac{1}{2}(\alpha(x)-\varepsilon) s^{2}-M,
$$

for all $\mathrm{s}$ in $R$ and for almost every $\mathrm{x}$ in $\Omega$. Then

$$
\begin{aligned}
\Phi(v) & \leq \frac{1}{2}\left(\|v\|^{2}-\lambda_{k}\|v\|_{2}^{2}-\int_{\Omega} \alpha(x) v^{2}\right)+\frac{\varepsilon}{2} \int v^{2}+C \\
& \leq \delta\|v\|^{2}+\frac{\varepsilon}{2}\|v\|_{2}^{2}+C
\end{aligned}
$$

Since $\mathrm{V}$ is of finite dimension, there exists $l>0$ such that $\|v\|^{2} \leq\|v\|_{2}^{2}$. So

$$
\Phi(v) \leq\left(\delta l+\frac{\varepsilon}{2}\right)\|v\|_{2}^{2}+C
$$

Consequently, it suffices to take $\varepsilon<-2 \delta l$ to conclude that $\Phi$ is coercive on $V$.

(v) $\Phi$ is weakely upper semi-continuous on $\mathrm{V}$. Indeed, $\mathrm{q}$ is a negative quadratic form on $\mathrm{V}$, then $\mathrm{q}$ is concave on $\mathrm{V}$, and since it's continuous, $\mathrm{q}$ is weakly upper semi-continuous on $\mathrm{V}$. Moreover, $\int F(x, u) \mathrm{dx}$ is weakely continuous on $\mathrm{H}$. So the result.

\section{References}

[1] Palais, R. and Smale, S., a generalized Morse theory., Bull. Amer. Math. Sos. 70, pp. 165-171, (1964).

[2] Cerami,G., Un criterio de esistenza per i punti critici su varietà ilimitate., Rc. Ist. Lomb. Sci. Lett. 112, pp. 332-336, (1978).

[3] Bartolo, P., Benci, V. and Fortunato, D., Abstract critical point theorems and applications to some nonlinear problems with strong resonance at infinity., J. Nonl. Anal. TMA 7, pp. 981-1012, (1983). 
[4] A.Hammerstein., Nichtlineare integralgleichungen nebst Anwendungen., Acta Math., 54, pp. 117-176, (1930).

[5] C.L.Dolph., Nonlinear integral equations of the Hammerstein type., Trans. Amer. Math. soc., 66, pp. 289-307, (1949).

[6] Jabri.,A critical point theorem without compactness and Applications. j. math. Analysis Applic. 90, pp. 64-71, (1982).

[7] Thews,K., A reduction method for some nonlinear Dirichlet problems., Nonlinear Anal. Theo.Methods App., 3, pp. 795-813, (1979).

[8] N.P.Các., On elliptic boundary value problems at double resonance., J.Math.Anal.Appl. 132, pp. 473-483, (1988).

[9] A. C Lazer, E. M. Landesman et D. R. Meyer., On saddle point problems in the calculus of variations, the ritz algorithm and monotone convergence., j. Math Anal. App. 52, pp. 594-614, (1975).

[10] Stepan A. Tersian., A minimax theorems and applications to nonresonance problems for semilinear equations, Nonlinear Analysis. Theory. Methods et Applications. vol. 10. No 7, pp. 651-668, (1986).

[11] Bates P. et Ekeland I, A saddle point theorem, in Differential equations, Academic Press, London, ((1980).

[12] Manasevich P. F.,A mini max theorem. j. math. Analysis Applic. 90, pp. 64-71, (1982). ..

[13] M.Moussaoui, Questions d'existence dans les problèmes semi-lineaires elliptiques. Thèse présentée en vue de l'obtention du grade de Docteur en Sciences, Université libre de Bruxelles, (1990 - 1991).

[14] Hafida Boukhrisse and M.Moussaoui, Critical point theorems. (To appear).

[15] J. Mawhin and M. Willem., Critical points of convex perturbations of some indefinite quadratic forms and semi-linear boundary value problems at resonance., Ann. Inst. Henry Poincaré., 3, pp. 431-453, (1986).

[16] S.Ahmad, A.C.Lazer, J.L.Paul., Elementary critical point theory and perturbations of elliptic boundary value problems at resonance., Indiana Univ. Math. J. 25, pp. 933-944, (1976). 
[17] M. S. Berger., Nonlinearity and Functional Analysis., Academic Press. New York/London, (1977).

[18] D.G.Costa., Topicos en anàlise nào-lineare e aplicaçoes às equaçoes diferenciais., Proc.E.L.A.M., Rio de Janeiro (1986), Springer Lect. Notes, à paraitre.

Recieved : September, 2001.

\section{Hafida Boukhrisse}

Mathematical Department

Faculty of Sciences

University Mohamed I

P. O. Box 524

60000 Oujda

Morocco

e-mail : boukhrisse@operamail.com

and

\section{Mimoun Moussaoui}

Mathematical Department

Faculty of Sciences

University Mohamed I

P. O. Box 524

60000 Oujda

Morocco

e-mail : moussaoui@sciences.univ-oujda.ac.ma 